\title{
Cholic acid mediates negative feedback regulation of bile acid synthesis in mice
}

\author{
Jia Li-Hawkins, ${ }^{1}$ Mats Gåfvels, ${ }^{2}$ Maria Olin, ${ }^{2}$ Erik G. Lund, ${ }^{1}$ Ulla Andersson, ${ }^{2}$ \\ Gertrud Schuster, ${ }^{2}$ Ingemar Björkhem, ${ }^{2}$ David W. Russell, ${ }^{1}$ and Gosta Eggertsen ${ }^{2}$
}

\footnotetext{
${ }^{1}$ Department of Molecular Genetics, University of Texas Southwestern Medical Center, Dallas, Texas, USA

${ }^{2}$ Department of Medical Laboratory Sciences and Technology, Huddinge University Hospital, Karolinska Institute, Huddinge, Sweden
}

\begin{abstract}
Cholesterol is converted into dozens of primary and secondary bile acids through pathways subject to negative feedback regulation mediated by the nuclear receptor farnesoid X receptor (FXR) and other effectors. Disruption of the sterol $12 \alpha$-hydroxylase gene (Cyp8b1) in mice prevents the synthesis of cholate, a primary bile acid, and its metabolites. Feedback regulation of the rate-limiting biosyn-

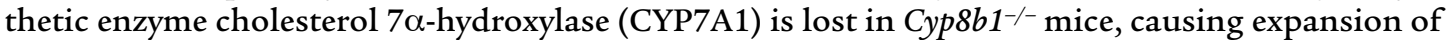
the bile acid pool and alterations in cholesterol metabolism. Expression of other FXR target genes is unaltered in these mice. Cholate restores CYP7A1 regulation in vivo and in vitro. The results implicate cholate as an important negative regulator of bile acid synthesis and provide preliminary evidence for ligand-specific gene activation by a nuclear receptor.
\end{abstract}

J. Clin. Invest. 110:1191-1200 (2002). doi:10.1172/JCI200216309.

\section{Introduction}

The synthesis and excretion of bile acids comprise the major pathway of cholesterol catabolism in mammals. Synthesis provides a direct means of converting cholesterol, a hydrophobic and insoluble molecule, into a watersoluble and readily excreted form, the bile acid. The biosynthetic steps that accomplish this transformation also confer detergent properties to the bile acid that are exploited by the body to facilitate the direct secretion of cholesterol from the liver and the solubilization of essential nutrients from the diet. These roles in the elimination of cholesterol are counterbalanced by the ability of bile acids to solubilize dietary cholesterol and to promote the delivery and accumulation of this sterol in the liver.

Bile acid synthesis is tightly regulated via a classical feedback mechanism to ensure that sufficient amounts

Received for publication June 28, 2002, and accepted in revised form August 13, 2002.

Address correspondence to: David W. Russell, Department of Molecular Genetics, University of Texas Southwestern Medical Center, 5323 Harry Hines Boulevard, Dallas, Texas 75390-9046, USA. Phone: (214) 648-2007; Fax: (214) 648-6899;

E-mail: david.russell@utsouthwestern.edu.

Jia Li-Hawkins and Mats Gåfvels contributed equally to this work.

Erik G. Lund's present address is: Merck and Co., Rahway, New Jersey, USA.

Conflict of interest: No conflict of interest has been declared. Nonstandard abbreviations used: cholesterol $7 \alpha$-hydroxylase (CYP7A1); farnesoid X receptor (FXR); short heterodimer partner (SHP); CYP7A1 promoter transcription factor (CPF); liver X receptor (LXR); hepatic nuclear factor (HNF); sterol $12 \alpha$ hydroxylase (CYP8B1); ileal bile acid binding protein (IBABP); sterol 27-hydroxylase (CYP27A1); oxysterol $7 \alpha$-hydroxylase (CYP7B1); bile salt export protein (BSEP); $\mathrm{Na}^{+}$-taurocholate cotransporting polypeptide (Ntcp). of cholesterol are catabolized to maintain homeostasis and provide adequate emulsification in the intestine. The accumulation of bile acids causes a decrease in the transcription of the cholesterol $7 \alpha$-hydroxylase gene (Cyp7a1), which encodes the first and rate-limiting enzyme in the major biosynthetic pathway (1). Conversely, the loss of bile acids results in enhanced transcription from this gene and a subsequent increase in biosynthetic output. Six members of the nuclear receptor family of transcription factors are known to regulate the expression of Cyp7a1 $(2,3)$. These include two proteins mediating repression of the gene, the farnesoid X receptor (also known as FXR, NR1H4) and the short heterodimer partner (SHP, NR0B2); three proteins that activate the gene, CYP7A1 promoter transcription factor (CPF, FTF, LRH-1, NR5A2), liver X receptor- $\alpha$ (LXR- $\alpha$, NR1H3), and hepatic nuclear factor- $4 \alpha$ (HNF- $4 \alpha$, NR2A1); and isoforms of the retinoid $X$ receptor (RXR, NR2B1-3) that act as essential partners for FXR and LXR.

When an organism is replete with bile acids, further synthesis is prevented by FXR, which upon binding bile acids (4-6) activates transcription of the Shp gene. SHP in turn binds to and inhibits the positively acting CPF receptor, causing a decrease in Cyp7a1 expression $(7,8)$. This regulatory circuit is less active when bile acids are depleted, allowing CPF and $\mathrm{HNF}-4 \alpha$ to stimulate transcription of Cyp $7 a 1$ and to restore the bile acid pool (9). In some species, including rats and mice, the expression of Cyp7a1 is increased by the accumulation of cholesterol in the liver (1). LXR and its ligands are responsible for this enhancement (10-13). Studies in knockout mice lacking LXR (13), FXR (14), or SHP $(15,16)$ confirm 
these observations and suggest the existence of additional regulatory mechanisms affecting bile acid synthesis that may be receptor independent.

Bile acids are remarkably diverse in structure and exist in many different forms within vertebrates. This complexity is evident in the mouse, in which chemical analyses have identified two primary bile acids, cholate and $\beta$-muricholate, which are the immediate products of the biosynthetic pathways, and seventeen secondary bile acids, which arise from the actions of the gut flora on primary bile acids (17). A long-standing question in the field is why so many different types of bile acids are present in a single organism (18). A diverse mixture of bile acids with different detergent properties may be required to ensure solubilization of the many hydrophobic nutrients encountered by the small intestine upon consumption of a varied diet. This prediction is supported by studies in which the effects of dietary bile acids on the solubilization of cholesterol are monitored (19). In this experimental situation, the fed bile acid accounts for a majority of the pool, and depending on its structure, either increases or decreases the amount of cholesterol absorbed by the small intestine. Alternatively, individual bile acids may differ in their ability to activate FXR and hence to regulate the synthesis of bile acids.

In the current studies we characterize a line of mice in which the capacity to synthesize the primary bile acid cholate is eliminated by targeted disruption of the sterol $12 \alpha$-hydroxylase gene (Cyp8b1). This alteration has some expected consequences for bile acid and cholesterol metabolism, including a change in the composition of the bile acid pool and a decrease in intestinal cholesterol absorption, as would be predicted from previous studies in mice with altered bile acid biosynthesis. Unexpectedly, the mutant mice have an expanded bile acid pool and an increased rate of bile acid synthesis. These phenotypic traits are traced to constitutive expression of cholesterol $7 \alpha$-hydroxylase. Since other FXR target genes are expressed normally in these animals, and the capacity to respond to cholate by repression of $7 \alpha$-hydroxylase remains in the Cyp $8 b 1^{-/-}$mice, the data suggest that cholate is responsible for feedback regulation of bile acid biosynthesis.

\section{Methods}

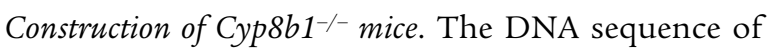
Cyp 861 obtained from the $129 \mathrm{SV} / \mathrm{J}$ strain of mice (20) was used to design primers for long-range PCR of $5^{\prime}$ and 3 '-flanking regions of the gene from genomic DNA isolated from embryonic stem cells of the $129 \mathrm{~Sv} / \mathrm{Ev}$ strain. The amplified DNAs, consisting of a $5.860-\mathrm{kb}$ long arm and a 3.011-kb short arm, were cloned on either side of a 3.143-kb lacZ gene (CLONTECH Laboratories, Palo Alto, California, USA) and a gene encoding resistance to geneticin (G418) obtained from the plasmid pPolIIIshort-neoPA-HSVTK (21). A single copy of the herpes simplex virus thymidine kinase gene obtained by PCR from the same plasmid was placed 3' of the Cyp8b1 short arm. Manipulation and assembly of DNA in vitro and propagation in E. coli were performed using standard methods (22).

Linearized targeting vector DNA was introduced into $2 \times 10^{7} 129 \mathrm{~Sv} /$ Ev mouse AB-1 embryonic stem cells by electroporation, and the resulting cells were subjected to plus-minus selection in DMEM supplemented with $15 \%$ (vol/vol) FCS, $250 \mu \mathrm{g} / \mathrm{ml} \mathrm{G} 418$, and $2 \mu \mathrm{M}$ ganciclovir. After 12-14 days, colonies were screened by Southern blotting of EcoRI-digested DNA using a radiolabeled genomic DNA fragment situated $3^{\prime}$ of the short arm of the targeting vector. DNA fragments from the wild-type $(8-\mathrm{kb})$ and targeted $(6.5-\mathrm{kb})$ alleles were identified in a single colony from among 1,296 screened, and the presence of the desired mutation was confirmed by PCR. Cells from this colony were injected into blastocysts from C57BL/6J females to produce 12 chimeric males with substantial contributions (>90\%) from the agouti stem cells. Several chimeric males transmitted the disrupted Cyp 861 gene through the germ line, producing 14 female and four male heterozygotes. Experiments were performed with mixed strain (C57BL/6J, 129Sv/Ev) descendants $\left(\mathrm{F}_{2}\right.$ and subsequent generations) of these animals. Routine genotyping of mice was performed on genomic DNA extracted from tails using multiplex PCR (see Figure $1 \mathrm{~b}$ ): wild-type allele $5^{\prime}$ primer I, CTTCCTCTATCGCCTGAAGCC; targeted allele 5' primer III, CCTGCAGCCAAGCTAGCTTGG; and common $3^{\prime}$ primer II, TGAGCTGACATGTGTTCC.

Bile acid composition and pool size. For gas chromatography/mass spectrometry analysis, bile was obtained from the gallbladders of wild-type and Cyp $8 b 1^{-/-}$mice and subjected to hydrolysis to remove conjugated taurine. Free bile acids were then identified and quantitated as described (23). For HPLC analysis, bile acids were isolated from the gallbladder, surrounding liver tissue, and small intestine of individual mice by extraction into ethanol, and then quantitated on a HewlettPackard (Palo Alto, California, USA) series 1050 system linked to a series 1100 refractive index detector (19).

Measurement of bile acid excretion rates. Animals were housed singly in fresh cages containing reduced amounts of wood chip shavings and fed a normal chow diet (no. 7001; Harlan Teklad Laboratory, Madison, Wisconsin, USA) ad libitum. After 3 days, stools were collected, dried, and weighed. A 1.0-g aliquot of stool from each animal was extracted with ethanol in the presence of a recovery standard, $\left[{ }^{14} \mathrm{C}\right]$ cholate (19). Extracted bile acids were quantitated using an enzymatic assay (24) and were expressed as $\mu \mathrm{mol} /$ day $/ 100 \mathrm{~g}$ body weight.

Cholesterol absorption and lipid excretion. Intestinal cholesterol absorption was measured using a fecal dual isotope ratio method (25). Total lipid and neutral sterol excretion rates were determined in stool collected as described above. Briefly, $0.5 \mathrm{~g}$ of feces was ground to a fine powder and saponified with ethanol $/ \mathrm{NaOH}$ at $120^{\circ} \mathrm{C}$ for 8 hours. Sterols were 
extracted into petroleum ether in the presence of 5 -cholestene as an internal recovery standard and quantitated by gas chromatography on a HewlettPackard 6890 series system. Peak heights of cholesterol, coprostanol, and cholestenone were compared with those derived from standards of known structure and concentration to estimate sterol mass. Fecal total lipid content was measured by extraction of 0.5 -g aliquots of stool with chloroform/methanol $(2: 1 \mathrm{vol} / \mathrm{vol})$ in the presence of $\left[{ }^{14} \mathrm{C}\right]$ triolein as an internal recovery standard. Extracted fats were dried in tared test tubes and their weights were determined on a microbalance.

De novo sterol and fatty acid synthesis. Rates of de novo sterol and fatty acid synthesis were measured using a $\left[{ }^{3} \mathrm{H}\right] \mathrm{H}_{2} \mathrm{O}$ incorporation assay (26). Each animal was administered $25 \mathrm{mCi}$ of $\left[{ }^{3} \mathrm{H}\right] \mathrm{H}_{2} \mathrm{O}$ intraperitoneally, followed by exsanguination 1 hour later. Blood, liver, small intestine, kidneys, spleen, and adrenal glands were collected and subjected to saponification. Sterols were extracted into petroleum ether and precipitated with digitonin, and incorporation of radiolabel was determined by scintillation counting. Aqueous phases from the petroleum ether extraction were acidified and extracted with hexane, and incorporation of radiolabel into fats was determined in the dried lipid by scintillation counting.

RNA measurements. Total RNA was extracted from frozen tissue using RNA Stat60 (Tel-Test "B" Inc., Friendswood, Texas, USA). Individual tissues from three to six mice per treatment group were isolated, and equal amounts of total RNA from each tissue were pooled for use as total RNA or for the purification of poly $(\mathrm{A})^{+}$RNA using an mRNA purification kit from Pharmacia (Piscataway, New Jersey, USA). Aliquots of total RNA $(15 \mu \mathrm{g})$ or poly $(\mathrm{A})^{+}(5 \mu \mathrm{g}) \mathrm{RNA}$ were analyzed by blotting using standard procedures (22). Hybridization probes were derived from mouse cDNAs encoding the indicated proteins, and in some cases were gifts of Jay Horton or David Mangelsdorf (University of Texas Southwestern Medical Center, Dallas, Texas, USA).

For real-time PCR analysis, total RNA from individual mice or from the pooled samples described above were treated with DNA-free (Ambion Inc., Austin, Texas, USA). Reverse transcription and PCR were performed with a SYBR Green RT-PCR kit and a PRISM 7900HT Sequence Detection System (Applied Biosystems, Foster City, California, USA). Aliquots (20 ng) of total RNA were used for each RT-PCR reaction. The sequences of the oligonucleotide primer pairs used to amplify the mRNAs were: CYP7A1, AGCAACTAAACAACCTGCCAGTACTA and GTCCGGATATTCAAGGATGCA; SHP, CAGCGCTGCCTGGAGTCT and AGGATCGTGCCCTTCAGGTA; ileal bile acid binding protein (IBABP), CAAGGCTACCGTGAAGATGGA and CCCACGACCTCCGAAGTCT; cyclophilin, TGGAGAGCACCAAGACAGACA and TGCCGGAGTCGACAATGAT; and GAPDH, TGTGTCCGTCGTGGATCTGA and CCTGCTTCACCACCTTCTTGAT.

Cholesterol $7 \alpha$-hydroxylase. Total protein extracts were prepared from frozen livers and subfractionated by differential centrifugation (27). Equal amounts of protein from the liver of each mouse in an experimental group were pooled prior to centrifugation. Aliquots $(150 \mu \mathrm{g})$ of subfraction protein were analyzed by immunoblotting (27). Cholesterol $7 \alpha$-hydroxalase enzyme activity was measured in microsomes using isotope dilution spectroscopy (28).

Diet studies. Mice of the indicated genotype $(n=3-4$ per experimental group) were housed one per cage and fed ad libitum either control powdered diet (no. 7001, Harlan Teklad Laboratory), or the same diet supplemented with $0.1 \%$ cholate, $0.25 \%$ cholate, $0.1 \%$ chenodeoxycholate, or $0.25 \%$ chenodeoxycholate (all wt $/ \mathrm{wt}$ ). Bile acids in the free-acid form were obtained from Sigma-Aldrich (St. Louis, Missouri, USA). After a 4-day feeding period, livers were dissected for preparation of total RNA as described above.

Hepatocytes. Hepatocytes were isolated from wild-type and $\mathrm{FXR}^{-1-}$ male adult mice. Animals were anesthetized with halothane and livers were perfused with $30 \mathrm{ml}$ Liver Perfusion Medium (Invitrogen Corp., San Diego, California, USA) followed by $30 \mathrm{ml}$ Liver Digestion Medium (Invitrogen Corp.), both at a flow rate of 2 $\mathrm{ml} / \mathrm{min}$. Isolated hepatocytes were resuspended in DMEM (Invitrogen Corp.) supplemented with 5\% ( vol/vol) FCS, 100 units/ml penicillin, and 100 units $/ \mathrm{ml}$ streptomycin, and placed in 6-well plates coated with collagen IV (Fisher Scientific Co., Pittsburgh, Pennsylvania, USA) at a density of $3.5 \times 10^{5}$ cells per well. After a 3 -hour incubation at $37^{\circ} \mathrm{C}$ in an atmosphere of $5 \% \mathrm{CO}_{2}$, the medium was changed to M199 (Invitrogen Corp.) supplemented with $0.1 \mu \mathrm{M}$ dexamethasone (Sigma-Aldrich), $0.1 \mu \mathrm{M} 3,3^{\prime}, 5$-triiodo-L-thyronine (T3, Sigma-Aldrich), $0.1 \mu \mathrm{M}$ insulin (SigmaAldrich), and the indicated (see figure legend) type and concentration of conjugated bile acid or FXR agonist GW4064. Cells were harvested 20 hours later and total RNA was isolated using an RNeasy kit (QIAGEN Inc., Valencia, California, USA) and an on-column DNase treatment. Relative mRNA levels were quantitated by real-time RT-PCR as described above.

\section{Results}

Deletion of the Cyp $8 b 1$ gene. Sterol $12 \alpha$-hydroxylase is a microsomal cytochrome $\mathrm{P} 450$ that catalyzes the addition of a hydroxyl group to carbon 12 of a number of sterol intermediates destined to become cholate in the bile acid biosynthetic pathways (Figure 1a). The enzyme is encoded by a gene (Cyp8b1) that lacks introns and is located on mouse chromosome $9 \mathrm{qF} 4$ (20). To disrupt Cyp861, a plasmid for use in homologous recombination was constructed in which the coding region of the gene was replaced with the E. coli $\beta$-galactosidase gene (lac Z) (Figure 1b). This DNA was electroporated into embryonic stem cells, which were then grown in the presence of ganciclovir and G418. Screening of 1,300 colonies identified a single isolate with the desired recombination event. Injection of this colony into blastocysts produced 12 chimeric males 

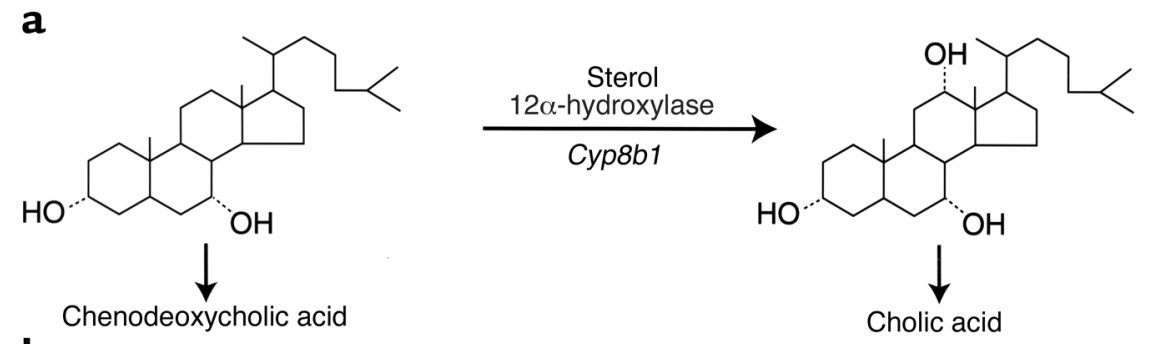

b

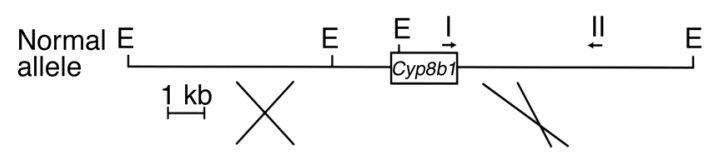

Targeting vector
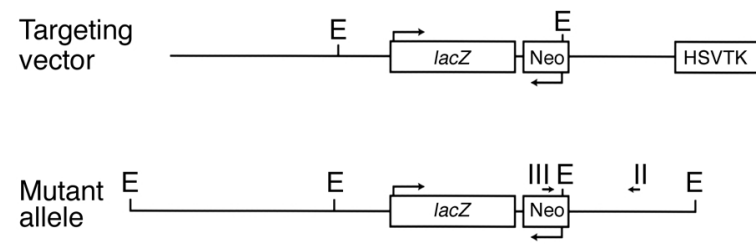

c

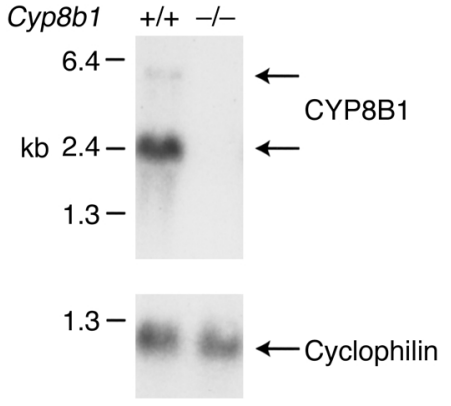

\section{Figure 1}

Deletion of mouse Cyp861. (a) Reaction catalyzed by CYP8B1, a microsomal cytochrome P450. The enzyme is quite promiscuous with respect to sterol substrate and acts on several $7 \alpha$-hydroxylated intermediates of the bile acid pathways, only one of which is depicted here. (b) Structures of normal Cyp861, the targeting vector used to replace the gene's coding sequence with that of E. coli $\beta$-galactosidase (lacZ), and the expected mutant allele following homologous recombination in embryonic stem cells. The diagnostic positions at which the restriction enzyme EcoRI (E) cleaves the DNAs are indicated. Boxes indicate a herpes simplex virus thymidine kinase gene (HSVTK) and a DNA encoding neomycin/geneticin resistance (Neo). Long arrows above or below the boxes indicate the directions of transcription for lacZ and the neomycin resistance genes. The locations of genotyping primers I, II, and III are shown by short arrows above the gene schematics. (c) Poly $(A)^{+}$RNA was isolated from the livers of animals $(n=6)$ of the indicated Cyp 861 genotype. Aliquots $(15 \mu \mathrm{g})$ of total RNA were subjected to blot hybridization using radiolabeled probes derived from the coding region of Cyp 861 and cyclophilin. The filter was exposed to $x$-ray film for 18 hours. The locations to which standards of known size migrated in the gel are indicated on the left of the autoradiogram.

(>90\% agouti), of which several transmitted the mutation through the germline. Crosses between Cyp8b1+/mice produced the expected number of wild-type, heterozygous, and homozygous offspring, and the survival rates of mice of each genotype were indistinguishable. CYP8B1 expression is limited to the liver (29), and analysis of RNA from this tissue revealed that the introduced mutation eliminated the encoding mRNA (Figure 1c).

Bile acid composition. The consequences of loss of Cyp8b1 on the types of bile acids present in the mutant mice were determined by gas chromatography/mass spectrometry (Figure 2). The primary bile acids cholate and $\beta$-muricholate together made up about $85 \%$ of the bile acids present in a male wild-type mouse (Figure 2). $\omega$-Muricholate accounted for approximately $6 \%$ of the pool, and the remainder consisted of small amounts of $\alpha$-muricholate (4\%), deoxycholate (1\%), chenodeoxycholate $(0.7 \%)$, and ursodeoxycholate $(0.6 \%)$. In contrast to this makeup, no cholate or its secondary metabolite, deoxycholate, were detected in Cyp $861^{-/-}$ mice (Figure 2), indicating that deletion of the gene eliminated CYP8B1 activity and that there were no compensating enzyme activities in the mutant mice. The fraction of the pool normally occupied by cholate was replaced mostly by increases in the muricholates. There were also substantial increases in the proportion of chenodeoxycholate (to 15\%) and ursodeoxycholate (to 9\%) in these animals (Figure 2). Similar alterations in the composition of bile acids were found in female knockout mice (data not shown).

Bile acid pool size and synthetic rate. Changes in the types of bile acids present in Cyp $861^{-/-}$mice were accompanied by an increase in the pool size, that is, the total mass of bile acids present in the animal (Figure $3 a$ ). The pool size in Cyp $861^{-1-}$ males was increased by $37 \%$ relative to that in wild-type controls, while that in Cyp $8 b 1^{-/-}$females was $20 \%$ larger than in the wild-type. Both of these increases were statistically significant $(P<0.05)$. Female wild-type mice typically have $35-40 \%$ larger bile acid pools than do males, as a consequence of increased output from the CYP7A1 pathway (30). This sexually dimorphic trait was

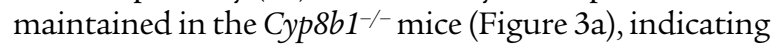
that male/female differences in bile acid synthesis cannot be ascribed to alterations in CYP8B1 activity.

Rates of bile acid synthesis in the liver were indirectly assessed by measuring the amount of bile acid excreted into the stool per day. As shown by the data in Figure 3b, this parameter was significantly increased in both male and female Cyp $861^{-/-}$mice. Since a majority of bile acids are synthesized via the CYP7A1 pathway in the mouse (19), these results suggested that the output from this highly regulated pathway was elevated in the knockout animals. 
Cholesterol absorption and excretion. Variations in bile acid pool size and composition affect the amount of cholesterol absorbed from the small intestine. In general, increases in the pool size and/or the content of hydrophobic bile acids in the pool cause increases in sterol absorption and vice versa. This relationship is illustrated in the sexes of wild-type mice; males absorb $44 \%$ of a bolus of cholesterol delivered intragastrically whereas females absorb 56\% (Figure 3c). The difference is attributable to an increase in the content of cholic acid in the female pool (30) and to an increase in the pool size in this sex (Figure 3a).

Both male and female Cyp $8 b 1^{-/-}$mice absorbed less cholesterol than their wild-type counterparts (Figure $3 c)$, despite having larger bile acid pool sizes. Furthermore, there was no statistical difference $(P=0.48)$ between the absorption values measured in knockout males (27\%) and those in knockout females (30\%), even though Cyp $8 b 1^{-1-}$ females had a larger pool size than did males (Figure 3a). These data suggested that of the two parameters of bile acid metabolism affecting solubilization and intestinal cholesterol absorption, composition was more important than pool size.

The observed differences in cholesterol absorption were confirmed by measurement of neutral sterol levels in the feces. As illustrated in Figure $3 \mathrm{~d}$, male and female Cyp $8 b 1^{-/-}$mice excreted significantly more neutral sterols (cholesterol, cholestanone, and coprostanol) than did wild-type mice. When the levels of total fecal lipid (fats plus neutral sterols) were determined, these too were found to be elevated in knockout males $(6.1 \% \pm 0.05 \%$ versus $5.0 \% \pm 0.04 \%, P=0.02)$ and females $(6.3 \% \pm 0.07 \%$ versus $5.5 \% \pm 0.04 \%, P=0.005)$. Approximately $2 \%$ of fecal lipid is composed of neutral sterols, while triacylglycerols and fatty acids account for about $98 \%$. The increases in total fecal lipid in the Cyp $8 b 1^{-/-}$mice thus indicate that the animals exhibited malabsorption of both sterols and fats (steatorrhea).

The decrease in absorption of dietary cholesterol in the Cyp $8 b 1^{-/-}$mice was accompanied by a significant increase in the synthesis of cholesterol by the liver. The de novo rate of hepatic sterol synthesis in male knockout mice was $2.3 \pm 0.4 \mu \mathrm{mol} / \mathrm{g}$ tissue $/ \mathrm{h}$ versus $1.0 \pm 0.2 \mu \mathrm{mol} / \mathrm{g}$ tissue $/ \mathrm{h}$ in wild-type controls. Cholesterol biosynthetic rates were not significantly different in other tissues of the mutant mice, including the kidney and adrenal gland, and triglyceride synthesis rates were unchanged in all tissues examined. The compensatory increase in hepatic sterol synthesis in response to sterol malabsorption in the gut maintained whole-body cholesterol homeostasis, since no statistically significant differences were detected in serum cholesterol and triglyceride levels, and the serum lipoprotein profiles of normal and mutant mice were superimposable (data not shown).

Cyp7a1 expression. The increases in bile acid pool size and excretion in the Cyp $8 b 1^{-/-}$mice suggested that output from the CYP7A1 pathway was increased. The data for Figures $4 \mathrm{a}$ and $4 \mathrm{c}$ showed that CYP7A1 mRNA and protein levels were substantially increased in both male

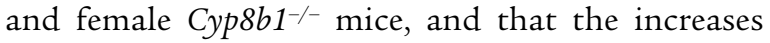
approached those seen in sterol 27-hydroxylase-deficient (Cyp27a1 $\left.{ }^{-/-}\right)$animals, which have a defect in bile acid synthesis (see below). Levels of CYP7A1 enzyme activity (Figure 4b) were also increased in the Cyp $8 b 1^{-/-}$ mice, although not to the degree of the mRNA and protein (Figure 4, a and c). No CYP7A1 mRNA or protein was detected in mice bearing a null mutation in the gene (Cyp7a1 ${ }^{-/-}$mice), and no changes in the levels of a control mRNA (cyclophilin) or microsomal protein (immunoglobulin heavy-chain binding protein; BIP) were measured in the mutant mice (Figure 4, a and c).

The expression levels of other enzymes involved in bile acid synthesis, including the CYP7B1 oxysterol $7 \alpha$-hydroxylase, the CYP27A1 sterol 27-hydroxylase, and the CYP39A1 oxysterol $7 \alpha$-hydroxylase, were

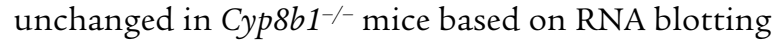
and immunoblotting (data not shown, and see below). As CYP7B1 and CYP39A1 expression are regulated by the nuclear receptor $\mathrm{HNF}-4 \alpha$ (31) these
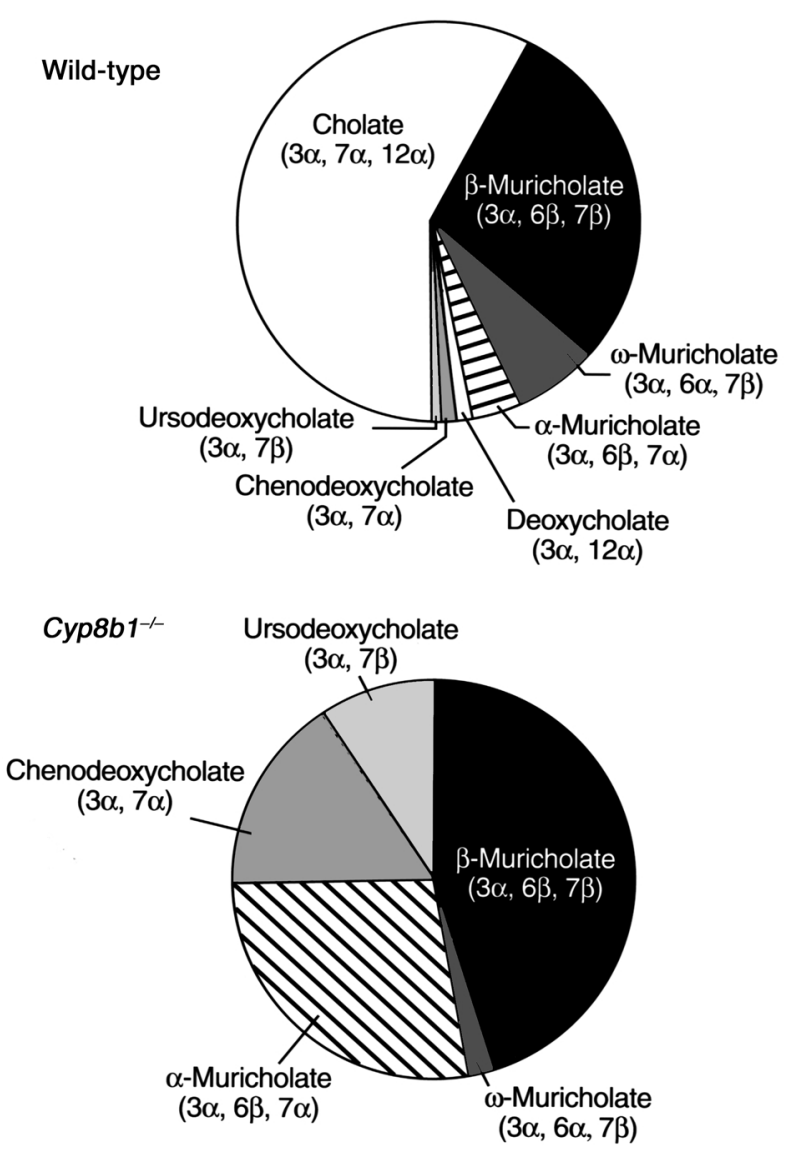

Figure 2

Composition of gallbladder bile in male wild-type and Cyp861-/- mice. Amounts of individual bile acids in the gallbladder bile of wild-type and Cyp861 $1^{-1-}$ mice were determined by gas chromatography/mass spectrometry and are indicated as a percentage of the entire pool. The position and stereochemistry of hydroxyl groups on the ring structures of each bile acid are indicated in parentheses. Similar results were obtained when the total bile acid pool was analyzed by HPLC. 

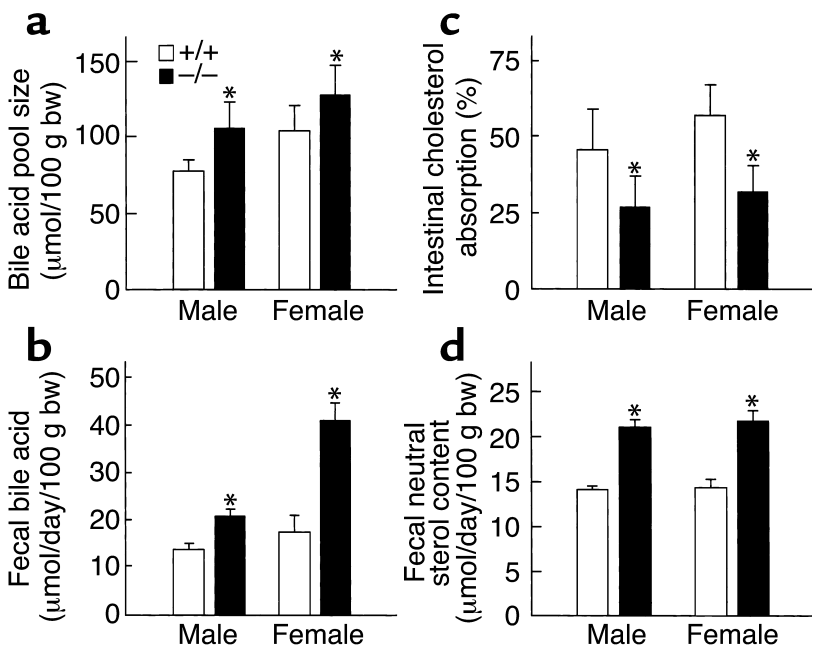

\section{d}

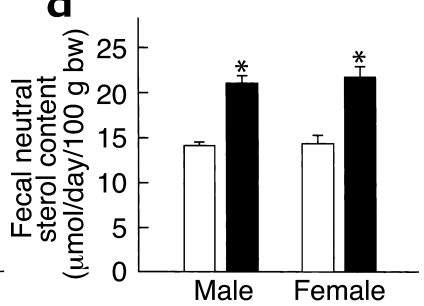

Figure 3

Bile acid pool sizes and excretion rates in wild-type and Cyp861-/mice. (a) The mass of bile acid in the enterohepatic circulation of normal and mutant male and female mice ( $n=6$ per group) was determined by extraction, purification, and quantitation by HPLC. Values (mean \pm SEM) determined in the Cyp861-/- mice were significantly different $(P=0.0037$ for males; $P=0.04$ for females by twotailed Student $t$ test) from those of normal controls. (b) Fecal bile acid excretion rates, a measure of hepatic bile acid synthesis, were determined as described in Methods. Excretion in Cyp861 $1^{-/-}$mice was significantly greater ( $P=0.003$ for males; $P=0.0002$ for females) than that in wild-type mice. (c) Radiolabeled cholesterol and a nonabsorbable recovery standard (sitostanol) were administered by gavage to male and female mice ( $n=6$ per group) of the indicated genotypes. The percentage of cholesterol absorbed was determined after extraction of sterols from the feces excreted from the animals over a 72-hour period. Absorption values measured in mutant mice were significantly smaller ( $P=0.02$ for males; $P=0.0007$ for females) than those in wild-type controls. (d) Fecal neutral sterols (cholesterol, coprostanol, and cholestanone) were quantitated by gas chromatography. Reduced absorption (see c) led to a significantly increased sterol excretion rate in Cyp861-1- mice $(P<0.0003$ for males and females). Bw, body weight.

findings suggested that the increase in CYP7A1 mRNA was specific and not related to a global change in hepatic transcription. Steady-state levels of CYP3A11 mRNA, which reflect the activity of the pregnane $\mathrm{X}$ receptor $(32,33)$, were the same in the livers of wild-type and Cyp $8 b 1^{-/-}$mice, and as expected were induced in Cyp27a1 $11^{-/}$mice (34).

FXR target gene expression. Mice with deficiencies in bile acid biosynthetic enzymes, such as the Cyp7a1 $1^{-/-}$ and Cyp27a1 $11^{-/}$animals, have reduced bile acid pool sizes and biosynthetic rates. These reductions decrease ligand availability and impair the ability of FXR to activate SHP, which in turn leads to activation of bile acid synthesis of the Cyp27a1 gene (Figure 4, a and c) $(7,23,35)$. The same mechanism could not explain the increased expression of the enzyme in the Cyp $8 b 1^{-/-}$ mice, since these animals have an elevated bile acid pool size and biosynthetic rate (Figure 3). Nevertheless, levels of hepatic SHP mRNA were reduced by about $50 \%$ in Cyp $8 b 1^{-1-}$ mice as judged by RNA blotting (Figure 5a), and by $46 \%$ in real-time PCR experiments (Figure $5 \mathrm{~b}$ ). In contrast to these results, the hepatic expression of other FXR target genes (36, 37 ), including the bile salt export protein (BSEP, also known as $\mathrm{ABCB} 11$ ) and the $\mathrm{Na}^{+}$-taurocholate cotransporting polypeptide (Ntcp, or SLC10A1), were unchanged in the Cyp $8 b 1^{-/-}$mice (Figure 5a). The mRNA level of organic anion transporter 2 (Oatp2, or SLC21A3) was no different, and FXR mRNA was elevated about twofold in the mutant mice. The latter two genes, Slc21a3 and Fxr, are dependent on the transcription factor HNF-1 $\alpha$ for expression (38), and thus these results indicated that the observed changes in SHP expression were not due to decreased HNF-1 $\alpha$ activity. Similarly, the amounts of the scavenger receptor BI (SR-BI) and CYP7B1 mRNAs, which are indirect and direct targets of HNF- $4 \alpha$, respectively (31), were unchanged (Figure 5a).

Levels of the FXR-responsive IBABP mRNA in the small intestine were also reduced by about $50 \%$ in the distal $20 \%$ of the small intestine (Figure $5 \mathrm{c}$ ). Data

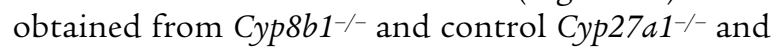
Cyp $7 \mathrm{a1}^{-/-}$mice confirmed that the expression of SHP was reduced in the small intestine when the bile acid pool size was changed (Figure $5 \mathrm{~b}$ ), and that the expression of BSEP and Ntcp mRNAs was variably affected in mice of these genotypes (Figure 5a).

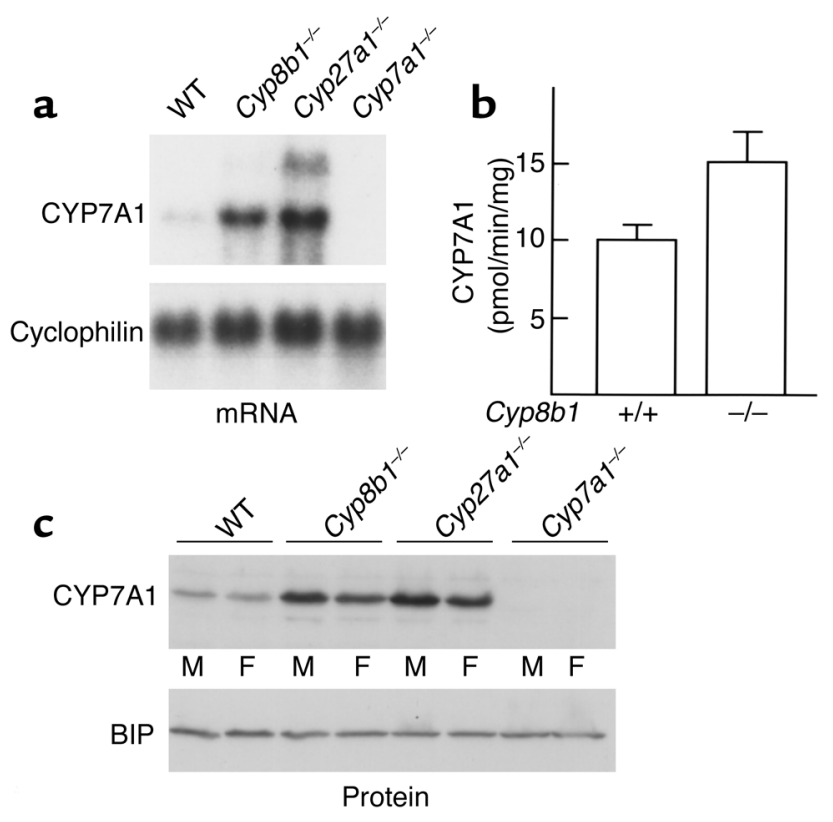

Figure 4

Expression of CYP7A1 in wild-type and knockout mice. (a-c) CYP7A1 mRNA, enzyme activity (expressed as pmol product per minute per mg microsomal protein), and protein were assessed by blotting, mass spectrometry, and immunoblotting, respectively, in male and/or female mice ( $n=6$ per group) of the indicated genotypes. Cyp27a ${ }^{-/-}$ mice have a reduced bile acid pool size and synthetic rate due to an induced mutation in the gene for sterol 27-hydroxylase, an enzyme involved in the synthesis of all primary bile acids. Cyp7a1--- mice served as negative controls in these experiments. BIP, immunoglobulin heavy-chain binding protein. 
Real-time PCR measurements of mRNAs encoding cholesterol supply pathway genes indicated that hydroxymethylglutaryl (HMG) CoA reductase mRNA was increased 1.5-fold and HMG CoA synthase mRNA was increased 1.4-fold in the knockout mice. These results were in agreement with the observed increase in hepatic de novo cholesterol synthesis in the Cyp8b1-/mice. The levels of LDL receptor mRNA were elevated 1.1-fold in the mutant mice, suggesting that most of the lost dietary cholesterol was replaced via the de novo biosynthetic pathway and not via receptor-mediated endocytosis of lipoproteins.

In a final experiment, the hepatic expression of approximately 400 genes involved in lipid metabolism was compared between wild-type and Cyp8b1-/- mice using cDNA microarray hybridization. The gene with the largest fold-increase (4.7-fold) was Cyp7a1, while the gene with the largest fold-decrease (to undetectable levels) was Cyp8b1. The chip hybridization results for other genes agreed with those obtained by RNA blotting (Figure $5 \mathrm{a}$ ). The outcomes from these three types of gene expression experiments revealed that changes in the bile acid composition and increases in the pool size in the Cyp8b1/- mice had differential effects on cholesterogenic and FXR target gene expression, impairing the transcription of some FXR-responsive genes (e.g., Shp and $I b a b p$ ) but leaving that of others (e.g., $A b c b 11$ and Slc10a1) neither increased nor decreased.

Cholate responsiveness in Cyp8b1-/- mice. To determine whether regulatory responses to bile acids had been lost in the Cyp $8 b 1^{-/-}$mice, groups of wild-type and mutant animals were fed chow diets supplemented with different amounts of cholate or chenodeoxycholate for 4 days. As expected, consumption of these diets altered the levels of the fed bile acid in the pool (see Figure 6 legend) and decreased de novo bile acid synthesis. The addition of $0.1 \%$ cholate to the diet caused a 70\% decrease in the level of CYP7A1 mRNA in wild-type mice, while the addition of $0.25 \%$ cholate caused a decline of greater than $98 \%$ (Figure 6). Chenodeoxycholate at $0.1 \%$ caused a $25 \%$ decrease in the mRNA, while diets with $0.25 \%$ caused a $90 \%$ decline. Similar results were obtained in the Cyp8b1-/mice (Figure 6). SHP mRNA levels correlated inversely with those of CYP7A1 in mice of both Cyp8b1 genotypes, and again, cholate appeared to be more efficacious than chenodeoxycholate in inducing SHP mRNA levels (Figure 6).

These data indicated that the capacity to respond to bile acids in the liver was intact in the Cyp $8 b 1^{-/-}$mice and that restoration of either cholate or chenodeoxycholate and their metabolites to the pool led to appropriate regulatory events. They also suggested that cholate was a more potent mediator of feedback regulation than chenodeoxycholate; however, this interpretation was tempered by the fact that the percentage of the fed bile acid in the pool differed between cholate and chenodeoxycholate, as did the amounts of other primary and secondary bile acids (Figure 6 legend).
Gene regulation in vitro. To examine the individual contributions of bile acids to regulation, we tested their abilities to activate different FXR target genes in vitro using hepatocytes prepared from wild-type and $\mathrm{FXR}^{-/-}$mice. These experiments used the taurine-conjugated forms of the bile acids and thus were designed to mimic the in vivo situation. As indicated by the data shown in Figure

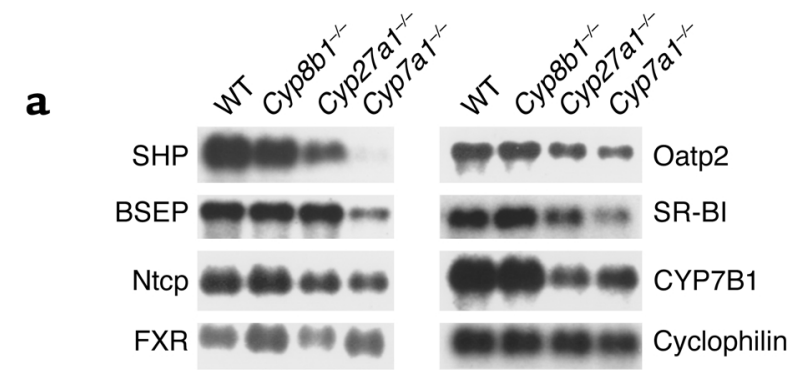

b
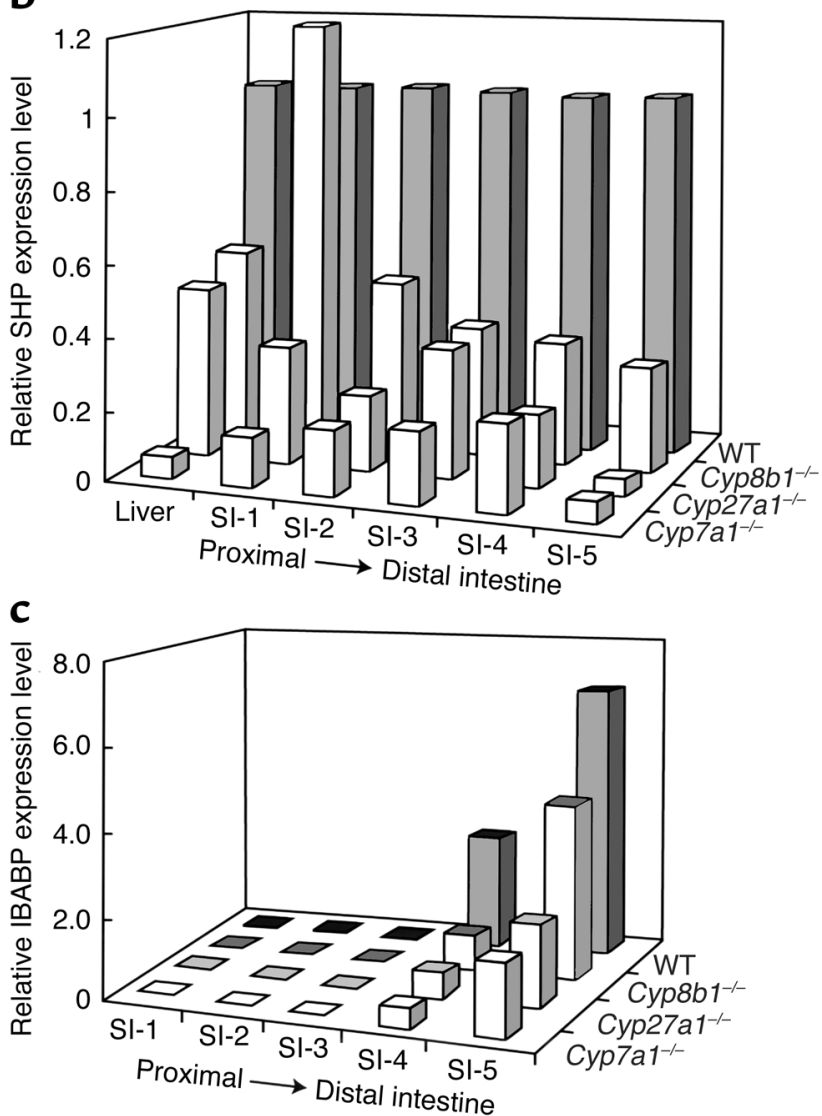

Figure 5

Gene expression in wild-type and Cyp861-/- mice. (a) mRNA levels for the indicated genes were determined by blotting in male mice ( $n=6$ per group) of the indicated genotypes. Autoradiographic exposure times varied from 7 to 18 hours. (b and $\mathbf{c}$ ) SHP and IBABP mRNA levels were quantitated in the livers and/or five intestinal segments (proximal to distal, S1-1 to S1-5) of male mice of the indicated genotype by real-time PCR in the presence of the intercalating dye SYBR Green. Levels of cyclophilin mRNA in each sample were used to normalize the results. The numbers shown are the averages of triplicate determinations for each mRNA and tissue. Oatp2, organic anion transporter 2 (also known as SLC21A3); SR-BI, scavenger receptor BI. 


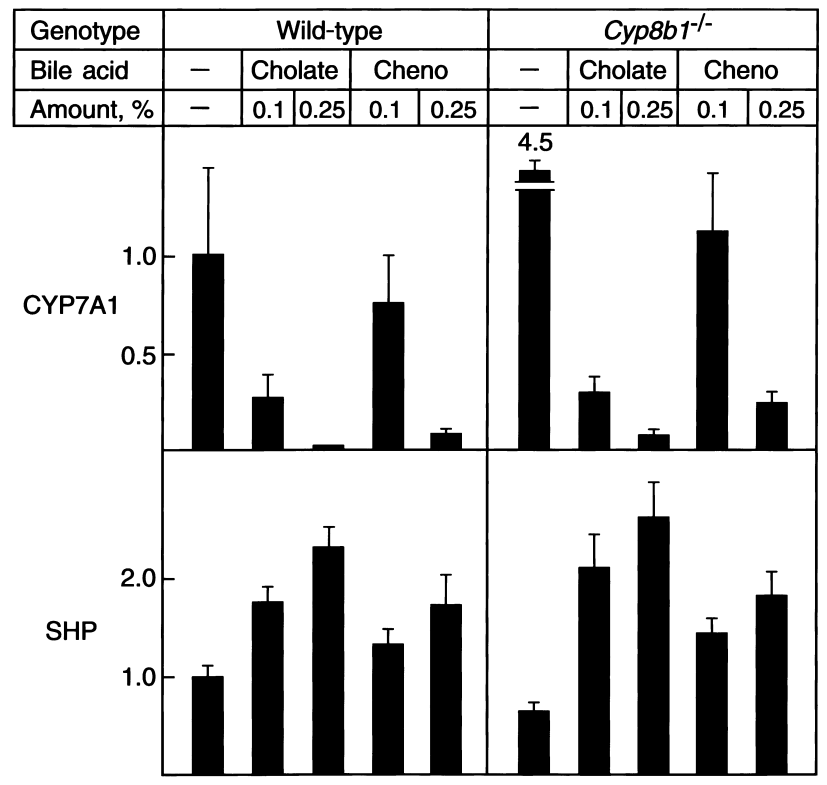

7a, taurocholic acid and the FXR agonist GW4064 (8) efficiently induced endogenous Shp expression in hepatocytes from wild-type mice. Chenodeoxycholate was less potent and less efficacious, whereas $\beta$-muricholate was inactive as an inducer. The levels of SHP mRNA were unchanged in response to bile acids or GW4064 in hepatocytes derived from $F X R^{-/-}$mice (Figure $7 \mathrm{~b}$ ).

In contrast to the response of SHP, levels of BSEP mRNA were induced equally well by cholate, chenodeoxycholate, and GW4064 in hepatocytes from wildtype mice (Figure $7 \mathrm{c}$ ). The addition of $\beta$-muricholate to these cells did not increase BSEP mRNA, and the responses of this mRNA to bile acids and GW4064 were reduced in the $\mathrm{FXR}^{-/-}$mice (Figure $7 \mathrm{~d}$ ).

Expression of the CYP7A1 and CYP8B1 mRNAs was lost upon isolation and plating of hepatocytes from mice of both FXR genotypes, and therefore we were unable to determine whether expression of SHP in response to cholate led to repression of these genes.

\section{Discussion}

The current results have implications for ligand-activated gene transcription mediated by nuclear receptors and for the regulation of bile acid metabolism. They

\section{Figure 7}

Regulation of gene expression by different bile acids in primary hepatocytes. Hepatocytes were isolated from the livers of wild-type and $F X R^{-1-}$ male mice and treated with the indicated concentrations of taurine-conjugated bile acids or the FXR-specific agonist GW4064 for 16 hours. Total RNA was isolated from the cells, and the levels of SHP and BSEP mRNA were quantitated by real-time RT-PCR. The numbers shown are the averages from three experiments and triplicate determinations for each mRNA. Error bars indicate SEM. TCA, taurocholic acid (circles); TCDCA, taurochenodeoxycholic acid (symbol X); T $\beta$ MCA, tauro- $\beta$-muricholic acid (squares); GW4064, 3-(2,6-dichlorophenyl)-4-(3'-carboxy-2-chloro-stilben-4-yl)oxymethyl-5-isopropyl-isoxazole (48) (triangles).

\section{Figure 6}

Responses to dietary bile acids in wild-type and Cyp861-/- mice. Male animals ( $n=4-5$ per genotype) were fed the indicated amounts of cholate or chenodeoxycholate (cheno) for a period of 4 days. mRNA levels for CYP7A1 and SHP were determined by real-time PCR. The numbers shown are the averages of triplicate determinations for each mRNA sample. Error bars indicate SEM. In wild-type mice fed $0.1 \%$ cholate, the composition of gallbladder bile was $15 \%$ muricholates, $78 \%$ cholate, $5 \%$ deoxycholate, and $2 \%$ other; in those fed $0.25 \%$ cholate it was $5 \%$ muricholates, $73 \%$ cholate, $15 \%$ deoxycholate, and $7 \%$ other. In those fed $0.1 \%$ chenodeoxycholate it was $58 \%$ muricholates, $13 \%$ cholate, $17 \%$ chenodeoxycholate, and $12 \%$ other, and in those fed $0.25 \%$ chenodeoxycholate it was $36 \%$ muricholates, $2 \%$ cholate, $47 \%$ chenodeoxycholate, and $15 \%$ other. Bile acid composition was similar in Cyp861-/- mice fed the same diets.

indicate that cholate, a primary bile acid in the mouse, is an in vivo mediator of negative feedback regulation of bile acid biosynthesis in this species. Loss of cholate, one of many potential ligands for the transcription factor FXR, from the bile acid pool causes a decrease in the hepatic expression of a negative regulatory factor (SHP), which in turn causes a loss of repression of the Cyp7a1 gene and an increase in biosynthetic output. This dysregulation appears to be specific, since transcription from other FXR-dependent genes in the liver (e.g., BSEP) is unaffected by the removal of cholate, and all expected regulatory responses are restored upon feeding cholate. Based on current understanding, one interpretation of these results is that the expression of SHP requires the binding and activation of FXR by cholate, whereas expression of BSEP by this receptor is accomplished by the binding of other bile acids. Alternatively, cholate may modulate SHP-independent negative feedback pathways that regulate CYP7A1 expression.

With respect to bile acid synthesis, these data underscore the important role of CYP8B1 in regulating the

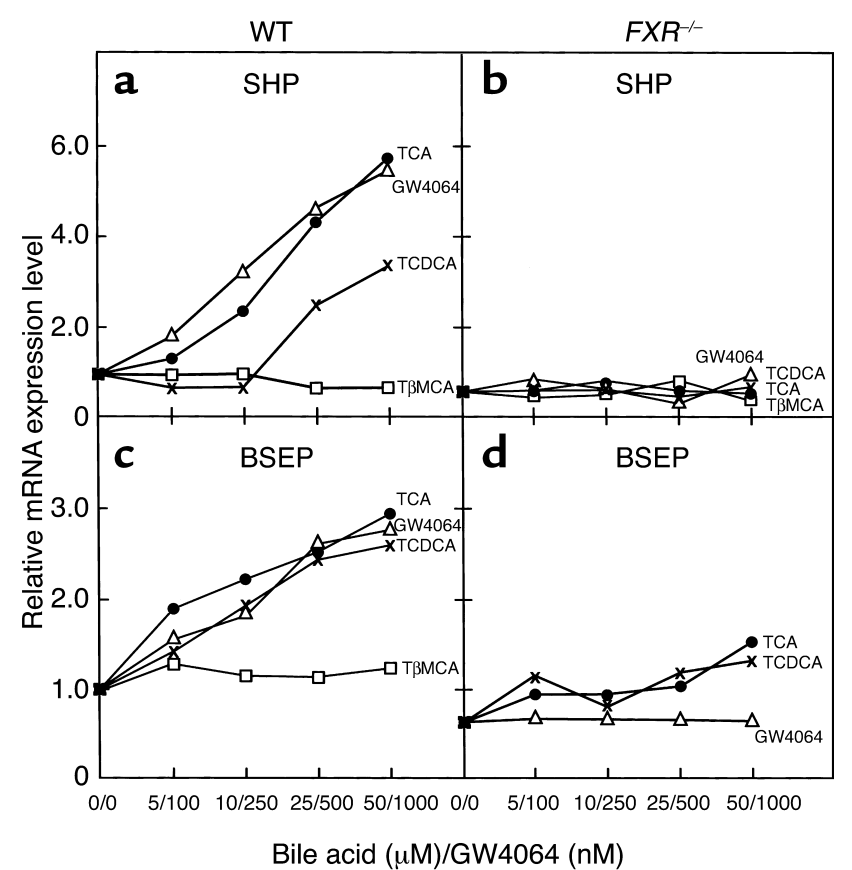


pathway and indicate why the expression of this enzyme is subject to negative feedback regulation. The inhibition of CYP8B1 expression by bile acids accomplishes three important goals. First, it acutely decreases the synthesis of a ligand required by FXR for feedback regulation. Second, it ensures that the system will gradually return to normal as the level of cholic acid is progressively reduced with each cycle of the enterohepatic circulation; the rate at which normalcy is reestablished becomes a function of the half-life of cholic acid in the system, which is among the shortest of all bile acid half-lives $(39,40)$. Third, as the half-life of a bile acid is largely dependent on factors in the gut rather than the liver (41), it allows one component tissue of the enterohepatic system to modulate a regulatory event occurring in another.

How might different bile acid ligands change the target specificity of FXR? Unique transcriptional responses could be mediated at the DNA level by the particular sequence or context of the response element (42). The FXR response element consists of two inverted repeats separated by one base pair (43), and variants of this sequence have been identified in the mouse SHP gene (AGGTGAATAACCT) $(7,8)$, the IBABP gene (AGGTGAATAACCT) (4), and the BSEP gene (GGGACATTGATCC) (36). The response elements in the SHP and $I B A B P$ genes are identical in sequence, and we note that the expression of both of these genes is decreased in the absence of cholate (Figure 5). Their response elements thus may be recognized by FXR in a ligand-dependent manner.

Bile acid-dependent responses could be accomplished at the protein level by the recruitment of different coregulators to a target gene following activation by a ligand. The ability of different bile acids to bind and activate FXR has been examined in transfection and binding experiments $(4-6,44)$. These studies show that chenodeoxycholate is a far better ligand than cholate, and in fact, in some $(4,5,44)$ but not all (6) transfection assays with monkey CV-1 and human HepG2 cells, cholate is inactive. Cholate was similarly unable to stimulate the binding of FXR to peptides derived from the 160 family of coactivator proteins. The inactivity of cholate in these assays contrasts with its very broad distribution in the bile of mammals (18), the elevated levels of CYP7A1 mRNA

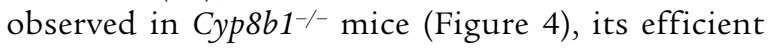
repression of bile acid synthesis in the whole animal (Figure 6), and the ability to preferentially stimulate SHP expression in primary hepatocytes (Figure 7). The discrepancies between the earlier findings and those presented here suggest there may be a hepatic coactivator protein that is missing from the immortalized cell lines and that is required for FXR to specifically stimulate SHP transcription.

Deletion of the Cyp8b1 gene prevents the synthesis of cholate in the mutant animal and at the same time eliminates all of the secondary bile acids derived from cholate. Chief among these is deoxycholate, which is the most abundant bile acid excreted in the stool of the mouse (17). This bile acid was toxic at concentrations over $10 \mu \mathrm{M}$ in the in vitro hepatocyte experiments reported in Figure 7, and for this reason, we cannot be certain that cholate is the only ligand responsible for the gene-specific regulation reported here. Nevertheless, it is clear that a $12 \alpha$-hydroxyl group must be present on the active metabolite for proper regulation of bile acid synthesis. If a secondary bile acid like deoxycholate is responsible for FXR-mediated activation of SHP, then the flora of the gut play an even more important role in bile acid and sterol metabolism than previously suspected (45).

The existence of multiple ligands for a single receptor extends the dynamic range over which transcription from a target gene can be regulated. The response to a particular ligand is unlikely to be all or none, as indicated by the results obtained with chenodeoxycholate

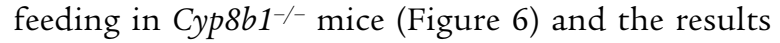
obtained with this ligand in hepatocytes (Figure 7). Chenodeoxycholate clearly is able to mediate feedback repression of CYP7A1 when elevated in the pool by feeding, and to induce SHP expression at higher concentrations in vitro, but in both of these systems it is less potent than cholate. This differential response is most clearly observed in Cyp8b1-/- mice maintained on normal chow. In these mice, chenodeoxycholate accounts for about $15 \%$ of the bile acid pool (Figure 2), yet this level is unable to suppress output from the Cyp7a1 gene. In contrast, BSEP mRNA expression is normal in the mutant mice (Figure 5a). These concentration-dependent effects suggest that the binding of more potent ligands like cholate to FXR amplifies the activation properties of the receptor.

An analogous situation exists in the case of the androgen receptor, which upon binding testosterone or dihydrotestosterone mediates different developmental programs involved in formation of the male phenotype. Mutations in the gene that synthesizes dihydrotestosterone block one of these pathways and typically lead to male pseudohermaphroditism (46); however, if the concentration of testosterone is elevated in dihydrotestosterone target tissues, the effects of the mutation are ameliorated and male development proceeds (47).

Studies in SHP knockout mice underscore the important role of this nuclear receptor in regulating bile acid synthesis and indicate that there are several FXR/SHP-independent pathways that negatively regulate the system $(15,16)$. The latter pathways may also respond to cholate, since the levels of CYP7A1 are con-

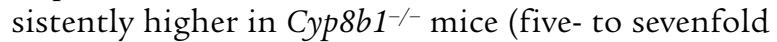
higher than in the wild-type) than they are in $\mathrm{SHP}^{-/-}$ mice (two- to threefold higher than in the wild-type). In vitro, the differential response to bile acids appears to be largely through FXR, since these ligands were inactive in hepatocytes lacking the receptor (Figure 7, b and d). How cholate affects the FXR-independent pathways that regulate bile acid synthesis in vivo is the subject of current research. 


\section{Acknowledgments}

We thank Kevin Anderson, Norma Anderson, Lorena Avila, and Scott Clark for excellent technical assistance; Jay Horton and Young-Ah Moon for help with in vivo sterol synthesis and hepatocyte preparation; Margrit Schwarz for GW4064; Frank Gonzalez for $F X R^{-/-}$mice; Alan Hofmann for discussions on the half-life of cholate; and Joe Goldstein and David Mangelsdorf for critical reading of the manuscript. This research was supported by grants from the NIH (HL-20948), the Keck Foundation, the Perot Family Foundation, and the Swedish Medical Research Council.

1. Russell, D.W., and Setchell, K.D.R. 1992. Bile acid biosynthesis. Biochemistry. 31:4737-4749.

2. Lu, T.T., Repa, J.J., and Mangelsdorf, D.J. 2001. Orphan nuclear receptors as eLiXiRs and FiXeRs of sterol metabolism. J. Biol. Chem. 276:37735-37738.

3. Chawla, A., Repa, J.J., Evans, R.M., and Mangelsdorf, D.J. 2001. Nuclear receptors and lipid physiology: opening the X-files. Science. 294:1866-1870.

4. Makishima, M., et al. 1999. Identification of a nuclear receptor for bile acids. Science. 284:1362-1365.

5. Parks, D.J., et al. 1999. Bile acids: natural ligands for an orphan nuclear receptor. Science. 284:1365-1368.

6. Wang, H., Chen, J., Hollister, K., Sowers, L.C., and Forman, B.M. 1999 Endogenous bile acids are ligands for the nuclear receptor FXR/BAR. Mol. Cell. 3:543-553.

7. Lu, T.T., et al. 2000. Molecular basis for feedback regulation of bile acid synthesis by nuclear receptors. Mol. Cell. 6:507-515.

8. Goodwin, B., et al. 2000. A regulatory cascade of the nuclear receptors FXR, SHP-1, and LRH-1 represses bile acid synthesis. Mol. Cell. 6:517-526.

9. Nitta, M., Ku, S., Brown, C., Okamoto, A.Y., and Shan, B. 1999. CPF: an orphan nuclear receptor that regulates liver-specific expression of the human cholesterol $7 \alpha$-hydroxylase gene. Proc. Natl. Acad. Sci. USA. 96:6660-6665.

10. Janowski, B.A., Willy, P.J., Devi, T.R., Falck, J.R., and Mangelsdorf, D.J. 1996. An oxysterol signaling pathway mediated by the nuclear receptor LXR $\alpha$. Nature. 383:728-731.

11. Lehmann, J.M., et al. 1997. Activation of the nuclear receptor LXR by oxysterols defines a new hormone response pathway. J. Biol. Chem. 272:3137-3140.

12. Forman, B.M., Ruan, B., Chen, J., Schroepfer, G.J., Jr., and Evans, R.M 1997. The orphan nuclear receptor $L X R \alpha$ is positively and negatively regulated by distinct products of mevalonate metabolism. Proc. Natl. Acad. Sci. USA. 94:10588-10593.

13. Peet, D.J., et al. 1998. Cholesterol and bile acid metabolism are impaired in mice lacking the nuclear oxysterol receptor LXRa. Cell. 93:693-704.

14. Sinal, C.J., et al. 2000. Targeted disruption of the nuclear receptor FXR/BAR impairs bile acid and lipid homeostasis. Cell. 102:731-744.

15. Kerr, T.A., et al. 2002. Loss of nuclear receptor SHP impairs but does not eliminate negative feedback regulation of bile acid synthesis. Dev. Cell. 2:713-720.

16. Wang, L., et al. 2002. Redundant pathways for negative feedback regulation of bile acid production. Dev. Cell. 2:721-731.

17. Schwarz, M., et al. 1996. Disruption of cholesterol $7 \alpha$-hydroxylase gene in mice. II. Bile acid deficiency is overcome by induction of oxysterol 7 $\alpha$-hydroxylase. J. Biol. Chem. 271:18024-18031.

18. Hofmann, A.F. 1999. Bile acid science (cholanology) at the dawn of a new millennium: past progress and the challenges for the future. In Bile acids in hepatobiliary disease. T.C. Northfield, H.A. Ahmed, R.P. Jazrawi, and P.L. Zentler-Munro, editors. Kluwer Academic Publishers. New York, New York, USA. 303-332.

19. Schwarz, M., Russell, D.W., Dietschy, J.M., and Turley, S.D. 1998. Marked reduction in bile acid synthesis in cholesterol $7 \alpha$-hydroxylase-deficient mice does not lead to diminished tissue cholesterol turnover or to hypercholesterolemia. J. Lipid. Res. 39:1833-1843.

20. Gafvels, M., et al. 1999. Structure and chromosomal assignment of the sterol $12 \alpha$-hydroxylase gene (CYP8B1) in human and mouse: eukaryotic cytochrome P-450 gene devoid of introns. Genomics. 56:184-196.

21. Mansour, S.L., Thomas, K.R., and Capecchi, M.R. 1988. Disruption of the proto-oncogene int- 2 in mouse embryo-derived stem cells: a general strategy for targeting mutations to non-selectable genes. Nature. 336:348-352.
22. Sambrook, J., and Russell, D.W. 2000. Molecular cloning: a laboratory manual. Cold Spring Harbor Laboratory Press. Plainview, New York, USA. 2400 pp.

23. Rosen, H., et al. 1998. Markedly reduced bile acid synthesis but maintained levels of cholesterol and vitamin D metabolites in mice with disrupted sterol 27-hydroxylase gene. J. Biol. Chem. 273:14805-14812.

24. Turley, S.D., and Dietschy, J.M. 1978. Re-evaluation of the $3 \alpha$-hydroxysteroid dehydrogenase assay for total bile acids in bile. J. Lipid. Res. 19:924-928.

25. Schwarz, M., Davis, D.L., Vick, B.R., and Russell, D.W. 2001. Genetic analysis of cholesterol absorption in inbred mice. J. Lipid Res. 42:1801-1811.

26. Jeske, D.J., and Dietschy, J.D. 1980. Regulation of rates of cholesterol synthesis in vivo in the liver and carcass of the rat measured using [3H]water. J. Lipid. Res. 21:364-376.

27. Li-Hawkins, J., Lund, E.G., Turley, S.D., and Russell, D.W. 2000. Disruption of the oxysterol $7 \alpha$-hydroxylase gene in mice. J. Biol. Chem. 275:16536-16542.

28. Andersson, U., et al. 1999. Thyroid hormone suppresses hepatic sterol $12 \alpha$-hydroxylase (CYP8B1) activity and messenger RNA in rat liver: failure to define known thyroid response elements in the gene. Biochim. Biophys. Acta. 1438:167-174.

29. Eggertsen, G., et al. 1996. Molecular cloning and expression of rabbit sterol $12 \alpha$-hydroxylase. J. Biol. Chem. 271:32269-32275.

30. Schwarz, M., Russell, D.W., Dietschy, J.M., and Turley, S.D. 2001. Alternate pathways of bile acid synthesis in the cholesterol $7 \alpha$-hydroxylase knockout mouse are not upregulated by either cholesterol or cholestyramine feeding. J. Lipid Res. 42:1594-1603.

31. Hayhurst, G.P., Lee, Y.-H., Lambert, G., Ward, J.M., and Gonzalez, F.J. 2001. Hepatocyte nuclear factor $4 \alpha$ (nuclear receptor 2A1) is essential for maintenance of hepatic gene expression and lipid homeostasis. Mol. Cell. Biol. 21:1393-1403.

32. Kliewer, S.A., et al. 1998. An orphan nuclear receptor pathway activated by pregnanes defines a steroid signaling pathway. Cell. 92:73-82.

33. Xie, W., et al. 2000. Humanized xenobiotic responses in mice expressing nuclear receptor SXR. Nature. 406:435-439.

34. Honda, A., et al. 2001. Side chain hydroxylations in bile acid biosynthesis catalyzed by CYP3A are markedly up-regulated in Cyp27-/- mice but not in cerebrotendinous xanthomatosis. J. Biol. Chem. 276:34579-34585.

35. Repa, J.J., et al. 2000. Disruption of the sterol 27-hydroxylase gene in mice results in hepatomegaly and hypertriglyceridemia: reversal by cholic acid feeding. J. Biol. Chem. 275:39685-39692.

36. Ananthanarayanan, M., Balasubramanian, N., Makishima, M., Mangelsdorf, D.J., and Suchy, F.J. 2001. Human bile salt export pump promoter is transactivated by the farnesoid $\mathrm{X}$ receptor/bile acid receptor. J. Biol. Chem. 276:28857-28865.

37. Denson, L.A., et al. 2001. The orphan nuclear receptor, shp, mediates bile acid-induced inhibition of the rat bile acid transporter, ntcp. Gastroenterology. 121:140-147.

38. Shih, D.Q., et al. 2001. Hepatocyte nuclear factor- $1 \alpha$ is an essential regulator of bile acid and plasma cholesterol metabolism. Nat. Genet. 27:375-382.

39. Danielsson, H., Eneroth, P., Hellstrom, K., Lindstedt, S., and Sjovall, J. 1963. On the turnover and excretory products of cholic acid and chenodeoxycholic acid in man. J. Biol. Chem. 238:2299-2304.

40. Hulzebos, C.V., et al. 2001. Measurement of parameters of cholic acid kinetics in plasma using a microscale stable isotope dilution technique: application to rodents and humans. J. Lipid Res. 42:1923-1929.

41. Carey, M.C. 1982. The enterohepatic circulation. In The liver: biology and pathobiology. Raven Press. New York, New York, USA. 429-465.

42. Hall, J.M., McDonnell, D.P., and Korach, K.S. 2002. Allosteric regulation of estrogen receptor structure, function, and coactivator recruitment by different estrogen responsive elements. Mol. Endocrinol. 16:469-486.

43. Forman, B.F., et al. 1995. Identification of a nuclear receptor that is activated by farnesol metabolites. Cell. 81:687-693.

44. Bramlett, K.S., Yao, S., and Burris, T.P. 2000. Correlation of farnesoid X receptor coactivator requirements and cholesterol $7 \alpha$-hydroxylase gene repression by bile acids. Mol. Genet. Metab. 71:609-615.

45. Hylemon, P.B., and Harder, J. 1998. Biotransformation of monoterpenes, bile acids, and other isoprenoids in anaerobic ecosystems. FEMS Microbiol. Rev. 22:475-488.

46. Wilson, J.D., Griffin, J.E., and Russell, D.W. 1993. Steroid 5 $\alpha$-reductase 2 deficiency. Endocrin. Rev. 14:577-593.

47. Mahendroo, M.S., Cala, K.C., Hess, D.L., and Russell, D.W. 2001. Unexpected virilization in male mice lacking steroid $5 \alpha$-reductase enzymes. Endocrinology. 142:4652-4662.

48. Maloney, P.R., et al. 2000. Identification of a chemical tool for the orphan nuclear receptor FXR. J. Med. Chem. 43:2971-2974. 